\title{
Conceivability and Modal Knowledge
}

Christopher Hill (2006) provides an account of modal knowledge that is set in a broader context of arguing against the view that conceivability provides epistemic access to the metaphysical modalities. His goal is "to identify the sources and forms of our knowledge of metaphysical possibility and metaphysical necessity" (205). ${ }^{1}$ The account proceeds in two stages. First, he contends that "metaphysical necessity and metaphysical possibility can be reductively explained in terms of the subjunctive conditional" (224). Second, he maintains that his reductive explanation of metaphysical necessity yields two tests for determining whether a proposition is metaphysically necessary and two corresponding tests for determining whether a proposition is metaphysically possible. ${ }^{2}$ Finally, Hill argues that his reductive account of the metaphysical modalities in conjunction with his account of modal knowledge underwrites the further conclusion that conceivability does not provide a reliable test for metaphysical possibility.

1. All page references in the text are to Hill (2006) unless otherwise indicated.

2. For an alternative attempt to reduce the metaphysical modalities to the subjunctive conditional and to provide an account of modal knowledge in terms of knowledge of subjunctive conditionals, see Williamson (2007). Williamson's account is critically evaluated in chapter 12 here. 
My focus is on Hill's two tests for determining the metaphysical modalities and how they bear on the view that conceivability provides epistemic access to metaphysical possibility. I argue that Hill's first test does not identify the source of our modal knowledge and, moreover, that an examination of the assumptions that motivate the test reveals that such knowledge does not require the employment of our cognitive mechanisms or procedures for evaluating subjunctive conditionals. I also argue that although the second test appears to provide an account of modal knowledge that does require the employment of our cognitive mechanisms or procedures for evaluating subjunctive conditionals, the appearances are misleading. A closer examination reveals that the second test collapses into the first, which does not require the employment of those cognitive mechanisms or procedures. Hence, Hill's reductive explanation of the metaphysical modalities in terms of the subjunctive conditional does not yield a reductive explanation of knowledge of metaphysical modality in terms of knowledge of subjunctive conditionals. Finally, I argue that his account of modal knowledge is at odds with his contention that conceivability does not provide epistemic access to metaphysical possibility.

\section{1}

Hill offers two arguments in support of the claim that metaphysical necessity is reducible to the subjunctive conditional. The first, which I call the A-argument, begins with Lewis's (1973) definition of a necessity operator in terms of the subjunctive conditional:

(A1) $\square \mathrm{A}=_{\mathrm{df}} \sim \mathrm{A}>\mathrm{A}$.

Hill's goal is to show that Lewis's operator expresses genuine metaphysical necessity. On Lewis's account, propositions containing $\square$ have the following truth conditions:

(A2) $\square \mathrm{A}$ is true at a possible world W just in case $\mathrm{A}$ is true at every possible world that is accessible from $\mathrm{W}$.

According to the standard picture,

(A3) If a proposition is metaphysically necessary, then it holds in all possible worlds. 
Hill (221), however, maintains:

(A4) The possible worlds that are accessible from $\mathrm{W}$ include all possible worlds. ${ }^{3}$

Therefore, it follows that

(A5) $\square \mathrm{A}$ is true at $\mathrm{W}$ just in case $\mathrm{A}$ is true at all possible worlds.

Since Lewis's operator captures the central feature of the standard picture, Hill concludes:

(A6) Lewis's operator expresses genuine metaphysical necessity.

Hill (223) notes that Lewis's definition of necessity can be restated as $\left(\mathrm{Al}^{*}\right)$ by using substitutional quantification

$$
\left(\mathrm{Al}^{*}\right) \quad \square \mathrm{A}={ }_{\mathrm{df}}(\Pi \mathrm{Q})(\mathrm{Q}>\mathrm{A}),
$$

where (ПQ) is the universal substitutional quantifier. His subsequent discussion employs $\left(\mathrm{A} 1^{*}\right)$.

Hill's second argument, which I call the B-argument, has two premises:

(B1) When we reflectively consider the familiar examples of metaphysical necessity, we find that they are always propositions that we are prepared to presuppose as valid in our subjunctive reasoning.

3. Hill offers the following supporting argument for (A4):

(S1) Suppose that $\mathrm{R}$ is a respect of comparison such that worlds not similar to the actual world in respect $\mathrm{R}$ are not accessible from the actual world.

(S2) This suggestion is refuted by the fact that there are nonvacuous counterfactuals that begin as follows: If the world were different in respect $\mathrm{R}, \ldots$

(S3) We regard such counterfactuals as having truth values.

(S4) We do not see them as vacuously true as is shown by the fact that we are prepared to give substantive reasons for accepting or rejecting them.

(S5) Therefore, we are prepared to entertain subjunctive conditionals that are arbitrarily different than the actual world. (221-222)

Hill goes on to extend the argument to show that where $\mathrm{W}$ is any possible world, all possible worlds are accessible from $\mathrm{W}$. 
(B2) When we consider the propositions that we presuppose as valid across subjunctive reasoning, we find that we are always prepared to regard them as metaphysically necessary.

(B3) Therefore, metaphysical necessity can be reductively explained in terms of the subjunctive conditional. (224)

The B-argument depends on the idea of presupposing a proposition as valid in subjunctive reasoning. Hill goes on to articulate three underlying assumptions that motivate his use of this idea.

Consider some proposition $\mathrm{P}$ that we treat as valid in subjunctive reasoning, such as that George W. Bush is a human being. Hill's underlying assumptions can be stated as follows:

(UA1) Our subjunctive reasoning reflects commitments to a range of propositions that have the following form: If $\mathrm{P}$, then (ПQ) (Q>P).

(UA2) These particular commitments derive from commitments to certain more general propositions such as:

(21) $(\forall \mathrm{x})(\forall \mathrm{K})$ (if $\mathrm{x}$ is a biological substance and $\mathrm{K}$ is a biological kind to which $\mathrm{x}$ belongs, then ( $\Pi \mathrm{Q})(\mathrm{Q}>\mathrm{x}$ is a biological substance that belongs to $\mathrm{K})$.

(UA3) Propositions like (21) are a priori propositions that are partially constitutive of certain of the concepts that occur in them-and in particular, of the subjunctive conditional. (226-227)

Hill (227-228) notes that the views articulated in (UA1)-(UA3) are closely related to Kripke's account of a posteriori necessities.

Kripke (1971, 153; quoted by Hill, 227) offers the following account of our knowledge of a posteriori necessities:

In other words, if $P$ is the statement that the lectern is not made of ice, one knows by a priori philosophical analysis, some conditional of the form "if $P$, then necessarily $P$." If the table is not made of ice, it is necessarily not made of ice. On the other hand, then, we know by empirical investigation that $P$, the antecedent of the conditional, is true-that this table is not made of ice. We can conclude by modus ponens:

$$
\begin{aligned}
& P \supset \square P \\
& \frac{P}{\square P}
\end{aligned}
$$


According to Kripke, our knowledge that necessarily $\mathrm{P}$, where $\mathrm{P}$ is some necessary a posteriori proposition, is based on an inference from two other propositions: (1) P; and (2) If P, then necessarily P. We know (1) a posteriori and (2) a priori. Hill (228) highlights the following parallel between his account and Kripke's. Where $\mathrm{P}$ is any necessary a posteriori proposition, such as that George W. Bush is a human being, Kripke holds that the following proposition can be known a priori:

(24) If $P$, then it is metaphysically necessary that $P$.

Hill, on the other hand, holds the following proposition can be known a priori:

(25) If $\mathrm{P}$, then (חQ) $(\mathrm{Q}>\mathrm{P})$.

Given $\left(\mathrm{Al}^{*}\right),(24)$ and (25) are analytically equivalent. Hill (228), however, adds the further claim, not found in Kripke's account, that propositions of the form (25) are definitional in character, following from more general principles, such as (21), that are partially constitutive of the subjunctive conditional. The fact that (25) follows from more general principles that are partially constitutive of the subjunctive conditional explains the a priori status of $(25) .{ }^{4}$

2

Hill contends that his reductive explanation of the metaphysical modalities yields two tests for determining whether a proposition is metaphysically necessary. The first, which I call the B-test, derives from the B-argument, whose central claim is that the class of propositions that we presuppose as valid in subjunctive reasoning is the same as the class of propositions that we regard as metaphysically necessary. Utilizing that claim, he argues that we can determine whether the proposition that $\mathrm{P}$ is metaphysically necessary by examining its role in subjunctive reasoning:

4. Hill offers the following remarks regarding constitutive propositions:

Suppose that the proposition that $P$ is partially constitutive of the concept $C$. It appears that all of the following are true. (i) We regard $P$ as available for use in justifications of other propositions that contain $C$, and for use in explanations of the truth of other propositions, but we do not regard it as desirable or even possible to provide a justification for $P$, or an explanation of the truth of $P \ldots$. (vi) We are committed to treating $P$ as operative in characterizing the belief systems of other agents, provided only that those agents are assumed to possess $C \ldots$. What I mean by this is that if we believe that another agent $A$ possesses the concept $C$, then we will think it prima facie appropriate to assume that $A$ believes that $P$. (207-208 n. 1) 
Suppose that when we analyze that role, we find that we are prepared to use $P$ in elaborating subjunctive suppositions whenever it is relevant to them, and that we never rely on propositions that are incompatible with $\mathrm{P}$ in elaborating subjunctive suppositions. In effect, we will have found that we presuppose that $\mathrm{P}$ is valid in subjunctive reasoning. Assuming that the foregoing claim about metaphysical necessity is correct, this result makes it reasonable to conclude that $\mathrm{P}$ is metaphysically necessary. (230)

According to the B-test, finding that we presuppose some proposition as valid in subjunctive reasoning provides a good reason to conclude that it is metaphysically necessary.

The B-test is of limited epistemic import. Although it may provide a procedure for identifying those propositions that we regard as metaphysically necessary, it tells us nothing about how we know those propositions that we regard as metaphysically necessary. In particular, the B-test does not show that we know those propositions by engaging our cognitive mechanisms or procedures for evaluating subjunctive conditionals.

The B-test exploits the following biconditional:

(BTl) We regard P as metaphysically necessary just in case we presuppose $\mathrm{P}$ as valid in subjunctive reasoning.

Suppose that we examine our subjunctive reasoning and discover:

(BT2) We presuppose $\mathrm{P}$ as valid in subjunctive reasoning.

According to Hill, we now have reason to conclude:

(BT3) P is metaphysically necessary.

Although (BT2) identifies $\mathrm{P}$ as a proposition that we presuppose as valid in subjunctive reasoning, it is silent about how we know the propositions that we presuppose as valid in subjunctive reasoning. More specifically, (BT2) does not entail or support the further conclusion that our knowledge of the propositions that we presuppose as valid in our subjunctive reasoning derives from our cognitive mechanisms or procedures for evaluating subjunctive conditionals. Therefore, the B-argument tells us nothing about the sources of our knowledge of metaphysical necessities.

Moreover, an examination of the assumptions that underlie the B-test reveals that they provide an account of our knowledge of metaphysical necessity in which our cognitive mechanisms or procedures for evaluating subjunctive conditionals 
play no role. Hill maintains that if $\mathrm{P}$ is a necessary proposition, then we can know a priori a proposition of the form:

(25) If $\mathrm{P}$, then (ПQ) $(\mathrm{Q}>\mathrm{P})$.

If we consider a necessary a posteriori proposition, such as

(P1) George W. Bush is a human being,

then the following proposition can be known a priori:

(P2) If George W. Bush is a human being, then (ПQ) ( $>>$ George W. Bush is a human being).

Moreover, according to Hill, (P2) follows from a more general a priori principle,

(21) $(\forall \mathrm{x})(\forall \mathrm{K})$ (if $\mathrm{x}$ is a biological substance and $\mathrm{K}$ is the biological kind to which $\mathrm{x}$ belongs, then (חQ) $(\mathrm{Q}>\mathrm{x}$ is a biological substance that belongs to $\mathrm{K}$ ),

that is partially constitutive of the subjunctive conditional. Given $\left(\mathrm{A} 1^{*}\right)$, one can infer:

(P3) Necessarily, George W. Bush is a human being

from (P1) and (P2).

The account of knowledge of necessary a posteriori propositions that emerges from the B-test, which I call the B-account, parallels Kripke's account. Knowledge of necessary a posteriori propositions, such as (P3), is inferential. It is based on knowledge of two premises: (P1) and (P2). Knowledge of (P1) is a posteriori, based on observations of Bush's physical features and behavior. (P2) is a consequence of (21), which is a proposition that is constitutive of the subjunctive conditional and that we can know a priori. Therefore, at the fundamental level, knowledge of a posteriori necessities does not involve either engaging our cognitive mechanisms or procedures for evaluating subjunctive conditionals or determining which propositions we presuppose as valid in our subjunctive reasoning. The only role played by subjunctive conditionals is that knowledge of (P2) derives from a priori principles that are constitutive of the concept of the subjunctive conditional. 
3

The second test, which I call the A-test, derives from the A-argument. According to it, we can determine whether the proposition that $\mathrm{P}$ is metaphysically necessary by evaluating a representative range of propositions that have the following form:

$$
\text { (30) } Q>P
$$

Hill (230) maintains: "There is no doubt that we can do this, for there is no doubt that we possess one or more cognitive mechanisms designed specifically to evaluate subjunctives." If we deploy these mechanisms and find that the representative propositions of the form (30) are all true, we will have good reason to believe

$$
\left(30^{*}\right) \quad(\Pi \mathrm{Q})(\mathrm{Q}>\mathrm{P})
$$

and, a fortiori, that $\mathrm{P}$ is metaphysically necessary. For example, Hill (231) maintains that if we wish to determine whether the proposition that $2 \times 3=6$ is metaphysically necessary, we can proceed by considering questions such as the following:

(Q1) Would it still be true that $2 \times 3=6$ if the only existing objects were abstract entities?

(Q2) Would it still be true that $2 \times 3=6$ if everything was in constant flux, with objects dissolving into new objects as soon as they were counted?

If the answers are all affirmative, then we have good reason to believe that $2 \times 3=6$ is metaphysically necessary.

Hill goes on to argue that our modal beliefs amount to knowledge. Here he appeals to the fact that subjunctive conditionals can be confirmed and disconfirmed by empirical evidence:

Now it seems to be the case that the subjunctive conditionals that we accept tend to be confirmed by the evidence that is relevant to them. If this impression is correct, then it is reasonable to regard our procedures for evaluating subjunctive conditionals as reliable, and by the same token, it is reasonable to regard our subjunctive beliefs as knowledge. (231-232)

Since we have empirical evidence that our procedures for evaluating subjunctive conditionals are reliable, we have reason to believe that beliefs based on those procedures constitute knowledge. 
The A-test appears to provide an account of modal knowledge that differs in a significant way from the B-account. The B-account of modal knowledge parallels Kripke's account. Our knowledge that necessarily $\mathrm{P}$, where $\mathrm{P}$ is some necessary a posteriori proposition, is based on an inference from two other propositions: (1) $P$, and (2) If $P$, then ( $\Pi Q)(Q>P)$. We know (1) a posteriori and (2) a priori, but neither our knowledge of (1) nor our knowledge of (2) requires engaging our cognitive mechanisms or procedures for evaluating subjunctive conditionals. The A-test, however, appears to provide an account of our modal knowledge that requires engaging our cognitive mechanisms or procedures for evaluating subjunctive conditionals. According to the A-test, we know propositions such as

$$
\left(30^{*}\right) \quad(\Pi \mathrm{Q})(\mathrm{Q}>\mathrm{P})
$$

by inductive generalization from a representative range of propositions that have the form

(30) $Q>$ P.

But, in order to know such propositions, we must employ our cognitive mechanisms or procedures for evaluating subjunctive conditionals. Therefore, the A-test appears to require the employment of our mechanisms or procedures for evaluating subjunctive conditionals.

Closer examination, however, reveals that the A-test does not require the employment of our cognitive mechanisms or procedures for evaluating subjunctive conditionals. Given the B-account of modal knowledge, the employment of our cognitive mechanisms or procedures for evaluating subjunctive conditionals is otiose; for anyone who is in the position to evaluate subjunctive conditionals of the form

$$
\text { (30) } \quad \text { Q }>\text { P }
$$

is also in a position to know a priori that they are true.

Consider Hill's application of the A-test to the necessary proposition that $2 \times 3=6$. According to that test, we examine instances of (30), such as

(I1) If the only existing objects were abstract entities, $2 \times 3=6$ would be true;

(I2) If everything was in constant flux, with objects dissolving into new objects as soon as they were counted, $2 \times 3=6$ would be true.

In order to evaluate (I1) and (I2), however, we must know whether the proposition that $2 \times 3=6$ is true. If, for example, we consider the following subjunctive conditionals 
(I3) If the only existing objects were abstract entities, the Goldbach Conjecture would be true;

(I4) If everything was in constant flux, with objects dissolving into new objects as soon as they were counted, the Goldbach Conjecture would be true;

we are not in a position to evaluate them since we don't know whether the Goldbach Conjecture is true.

Once we recognize that the A-test is applicable only if we know the truth value of the proposition in question, we can also see that we need not engage our cognitive mechanisms or procedures for evaluating subjunctive conditionals in order to apply it. Consider a necessary proposition, such as

(P1) George W. Bush is a human being,

and assume that we know that it is true. According to Hill, if $\mathrm{P}$ is a necessary proposition, then we can know a priori a proposition of the form

(25) If $\mathrm{P}$, then (חQ) $(\mathrm{Q}>\mathrm{P})$.

Hence, we can know a priori

(P2) If George W. Bush is a human being then (חQ) (Q $>$ George W. Bush is a human being).

(P2) is a consequence of the more general a priori principle

(21) $(\forall x)(\forall K)$ (if $x$ is a biological substance and $K$ is the biological kind to which x belongs, then ( $\Pi Q)(Q>x$ is a biological substance that belongs to $K$ ),

which is constitutive of the subjunctive conditional and can be known a priori. Hence, anyone who possesses the concept of the subjunctive conditional and knows that $(\mathrm{P} 1)$ is true can know via inference from (P1) and (P2) that

(P4) (ПQ) (Q> George W. Bush is a human being).

There is no need to evaluate a range of propositions of the form

$$
\text { Q }>\text { George W. Bush is a human being }
$$

and to inductively infer (P4) on that basis. Moreover, anyone who possesses the concept of the subjunctive conditional and knows that (P1) is true can know 
propositions of the form (P5) by inference from (P4). Therefore, where $\mathrm{P}$ is a necessary proposition, one who knows that $\mathrm{P}$ is true can know both general propositions of the form

\section{$\left(30^{*}\right) \quad(\Pi \mathrm{Q})(\mathrm{Q}>\mathrm{P})$}

as well as their instances, without employing the cognitive mechanisms or procedures that we use to assess subjunctive conditionals. ${ }^{5}$ Their employment is otiose.

One might object that my assessment of the significance of the A-test is unduly pessimistic. Even if the B-account of modal knowledge indicates that it is unnecessary to employ our cognitive mechanisms or procedures for evaluating subjunctive conditionals in order to know propositions of the form

$$
\mathrm{Q}>\mathrm{P}
$$

it does not follow that those mechanisms or procedures cannot be employed to arrive at such knowledge. We may have here a case of epistemic overdetermination; that is, two different, but independent, routes to knowledge of the metaphysical modalities.

Although epistemic overdetermination remains a possibility on Hill's account of knowledge of metaphysical necessities, there are two reasons for doubting that

5. I have argued that the only role played by subjunctive conditionals in Hill's account of modal knowledge is that general principles, such as (21), are alleged to be constitutive of the concept of the subjunctive conditional. But even this role seems dispensable given the close relationship between Hill's first test and Kripke's account. Where $P$ is any necessary a posteriori proposition, Kripke holds that the following proposition is knowable a priori:

(24) If $\mathrm{P}$, then it is metaphysically necessary that $\mathrm{P}$,

whereas Hill holds the following proposition is knowable a priori:

(25) If $\mathrm{P}$, then (חQ) $(\mathrm{Q}>\mathrm{P})$.

For Hill, propositions of form (25) are knowable a priori because they follow from more general principles, such as $(21)$, that are alleged to be constitutive of the concept of the subjunctive conditional. One who favors Kripke's account has available a similar strategy. He or she could maintain that propositions of the form (24) are knowable a priori because they follow from more general principles, such as

$\left(21^{*}\right)(\forall \mathrm{x})(\forall \mathrm{K})$ (if $\mathrm{x}$ is a biological substance and $\mathrm{K}$ is the biological kind to which $\mathrm{x}$ belongs, then $\square$ ( $\mathrm{x}$ is a biological substance that belongs to $\mathrm{K})$,

that are constitutive of the concept of metaphysical necessity. Hill's appeal to subjunctive conditionals adds a layer of theory that offers no increase in explanatory power over the view inspired by Kripke's account. 
there is an independent route to such knowledge via our procedures for evaluating subjunctive conditionals. First, there is a significant question that can be raised with respect to Hill's argument in support of the claim that beliefs about metaphysical necessity generated by the evaluation of subjunctive conditionals amount to knowledge. Second, there is a significant gap in Hill's account of how we acquire knowledge of metaphysical necessities via the exercise of our procedures for evaluating subjunctive conditionals.

Hill's argument (231-232) in support of the claim that our beliefs about metaphysical necessity amount to knowledge can be stated as follows:

(K1) Claims of metaphysical necessity are equivalent to generalized subjunctive conditionals.

(K2) Subjunctive conditionals can be confirmed and disconfirmed by empirical evidence.

(K3) The subjunctive conditionals that we accept tend to be confirmed by the empirical evidence that is relevant to them.

(K4) Therefore, it is reasonable to regard our procedures for evaluating subjunctive conditionals as reliable and our subjunctive beliefs as knowledge.

The key premise in the argument is (K3). For example, the subjunctive conditional

$\left(I^{*}\right) \quad$ If I hadn't given her the medicine, she wouldn't have recovered

gives rise to the indicative prediction

$\left(\mathrm{IP}^{*}\right)$ If medication is withheld in similar cases, the patients will not recover.

So cases of patients who do not recover because medication is withheld confirm $\left(\mathrm{IP}^{*}\right)$.

The subjunctive conditionals that are involved in the evaluation of metaphysically necessary propositions, however, do not appear to be confirmed by empirical evidence. Consider, for example, the subjunctive conditionals that, according to Hill, are relevant to the confirmation of the necessary proposition that $2 \times 3=6$ :

(I1) If the only existing objects were abstract entities, $2 \times 3=6$ would be true; and

(I2) If everything was in constant flux, with objects dissolving into new objects as soon as they were counted, $2 \times 3=6$ would be true; 
which give rise to the indicative predictions:

(IP1) If all concrete objects are destroyed, then $2 \times 3=6$ is true; and

(IP2) If everything is placed in constant flux, with objects dissolving into new objects as soon as they were counted, $2 \times 3=6$ is true.

It is unclear what empirical evidence, if any, supports (IP1) and (IP2). If we consider

(P1) George W. Bush is a human being,

the situation is worse. We lack an account of (a) the subjunctive conditionals relevant to confirming (P1), (b) the relevant indicative predictions that derive from those subjunctive conditionals, and (c) the empirical evidence that confirms those predictions. Hence, at best, (K3) remains questionable for the case of metaphysically necessary propositions.

Even if (K3) can be substantiated in the case of metaphysically necessary propositions, a significant gap remains in Hill's account of our knowledge of metaphysical necessities. On his view, a metaphysically necessary proposition that $\mathrm{P}$ is analytically equivalent to a universally quantified proposition of the form

$$
\left(30^{*}\right) \quad(\Pi \mathrm{Q})(\mathrm{Q}>\mathrm{P}) \text {. }
$$

His argument, if sound, establishes that we have knowledge of the premises that constitute the basis of our alleged (inductive) inferential knowledge that $\left(30^{*}\right)$ via the exercise of our procedures for evaluating subjunctive conditionals. But Hill has not provided any indication of how we move from knowledge of such premises to knowledge of metaphysically necessary propositions. His account is silent about (a) the content of the inferential principle (or principles) that mediate the transition from premises of the form

$$
\text { (30) }(\mathrm{Q}>\mathrm{P})
$$

to conclusions of the form

$$
\left(30^{*}\right) \quad(\Pi \mathrm{Q})(\mathrm{Q}>\mathrm{P})
$$

and (b) how we know those principles. Therefore, the case for overdetermination is, at best, premature. 
4

I have argued that the A-test does not require the employment of our cognitive mechanisms or procedures for evaluating subjunctive conditionals. Given the $\mathrm{B}$-account of modal knowledge, their employment is otiose. Moreover, as I shall now argue, the reason why Hill fails to recognize that their employment is otiose is that he fails to distinguish two different targets of an account of modal knowledge. This failure also mitigates his negative assessment of the view that conceivability provides epistemic access to the metaphysical modalities.

There are two different potential targets for an account of modal knowledge. In order to locate them precisely, let us introduce the following distinctions:

(A) $\mathrm{S}$ knows the truth value of $\mathrm{p}$ just in case $\mathrm{S}$ knows that $\mathrm{p}$ is true or $\mathrm{S}$ knows that $\mathrm{p}$ is false.

(B) $\mathrm{S}$ knows the general modal status of $\mathrm{p}$ just in case $\mathrm{S}$ knows that $\mathrm{p}$ is a necessary proposition (i.e., necessarily true or necessarily false) or $S$ knows that $\mathrm{p}$ is a contingent proposition (i.e., contingently true or contingently false).

(C) $\mathrm{S}$ knows the specific modal status of $\mathrm{p}$ just in case $\mathrm{S}$ knows that $\mathrm{p}$ is necessarily true or $S$ knows that $p$ is necessarily false or $S$ knows that $\mathrm{p}$ is contingently true or $\mathrm{S}$ knows that $\mathrm{p}$ is contingently false.

An account of modal knowledge can have as its target either knowledge of the general modal status of a proposition or knowledge of its specific modal status.

The goal of the A-test is to provide an account of knowledge of propositions of the form

\section{$\left(30^{*}\right) \quad(\Pi Q)(\mathrm{Q}>\mathrm{P})$,}

which, by $\left(\mathrm{A} 1^{*}\right)$, are analytically equivalent to propositions of the form $\square \mathrm{P}$. Hence, the target of the A-test is knowledge of the specific modal status of necessary propositions. But, as Kripke's discussion of the lectern example highlights, knowledge of the specific modal status of a necessary proposition is the conjunction of knowledge of its truth value and knowledge of its general modal status. Moreover, on Kripke's account, knowledge of the general modal status of a necessary proposition is more fundamental than knowledge of its specific modal status. Knowledge of the specific modal status of a necessary proposition is based on an inference from knowledge of its general modal status and knowledge of its truth value. The B-account of modal knowledge, which parallels Kripke's account, has as its target knowledge of the general modal status of necessary propositions. Since it provides an account of knowledge of the general modal 
status of necessary propositions in terms of constitutive a priori principles, it is unnecessary to employ our cognitive mechanisms or procedures for evaluating subjunctive conditionals in order to know propositions of the form $\left(30^{*}\right)$. Anyone who is in the position to evaluate subjunctive conditionals of the form

$$
\text { (30) } Q>P
$$

is also in a position to know a priori that they are true.

Hill's failure to distinguish the two different targets of an account of modal knowledge also mitigates his pessimistic assessment of the relationship between conceivability and the metaphysical modalities. He contends that it is a mistake to think that "conceivability is somehow the foundation of our epistemic access to these metaphysical modalities" (205). More specifically, his goal is to argue that the following principle, which I call the Conceivability Principle, is false: If it is within our power to conceive coherently of its being the case that $\mathrm{P}$, then it is metaphysically possible that $\mathrm{P}$.

Hill offers the following articulation of the concept of coherent conceiving:

if it is true that we can coherently conceive of its being the case that $P$, then it must also be true (a) that we are able to entertain the proposition that $P$, (b) that the proposition that $P$ is compatible with the laws of logic, (c) that the proposition that $P$ is compatible with the laws of mathematics, and (d) that the proposition that $P$ is compatible with all of the a priori principles that are constitutive of the relevant concepts,... Finally,... in addition to being necessary conditions of our being able to conceive coherently of its being the case that $P,(a)-(d)$ are also sufficient for us to have this ability. (207)

Since Hill holds that the propositions in conditions (b)-(d) are both a priori and cognitively robust (i.e., they play an essential role in the edifice of human knowledge), he labels them AR-propositions.

Hill's (208) account of coherent conceiving has the consequence that "we can coherently conceive of the truth of any entertainable proposition that is not precluded by AR-propositions." Assuming that an a priori proposition is one that can be known a priori and that an a posteriori proposition is one that can be known only a posteriori, it follows that any entertainable a posteriori proposition is coherently conceivable. In particular, if we consider examples of necessary a posteriori propositions, such as

(P1) George W. Bush is a human being; and

(P6) Hesperus is identical with Phosphorus; 
their negations are coherently conceivable. ${ }^{6}$ We can coherently conceive of the truth of both not-(P1) and not-(P6) because we can entertain them and their truth is compatible with all propositions that are knowable a priori.

In order to assess the relationship between coherent conceiving and possibility, Hill (209-210) distinguishes two forms of possibility:

(CP) It is conceptually possible that $\mathrm{P}$ if the proposition that $\mathrm{P}$ is compatible with all of the propositions that count as AR-propositions; and

(MP) It is metaphysically possible that $\mathrm{P}$ if the proposition that $\mathrm{P}$ is compatible with all AR-propositions and, in addition, with a large and heterogenous class of propositions that enumerate the essential properties of substances and kinds.

Hill (211) contends that coherent conceiving is sufficient for conceptual possibility but not for metaphysical possibility since "the conditions that define coherent conceiving are much less restrictive than the conditions that define metaphysical possibility." It is possible for a proposition to satisfy the former without satisfying the latter because the former requires only compatibility with all AR-propositions, but the latter requires, in addition, compatibility with all propositions that state the essential properties of substances and kinds (call them E-propositions).

Hill's argument against the Conceivability Principle is based on three contentions. First, coherent conceiving requires compatibility only with AR-propositions, that is, propositions that are a priori and cognitively robust. Second, metaphysical possibility requires compatibility with both AR-propositions and E-propositions. Third, E-propositions are not a priori. Consider an E-proposition, such as

(P1) George W. Bush is a human being.

Since (P1) is not an a priori proposition, it is not an AR-proposition. Since (P1) is not an AR-proposition, not-(P1) is compatible with all AR-propositions and, as a consequence, is coherently conceivable. Therefore, according to the Conceivability Principle, it is metaphysically possible that not-(P1), which entails that it is not necessarily true that (P1). Since (P1) is necessarily true, Hill's argument establishes that coherent conceiving is not a reliable guide to the specific modal status of E-propositions.

The view that conceivability provides epistemic access to the metaphysical modalities is ambiguous. It can be understood in two ways:

6. I assume here, following Hill, that Kripke is correct about the epistemic status of these propositions. The issue is controversial. In particular, some maintain that (P6) is a priori. For a discussion of some of the complexities generated by Kripke's examples, see Casullo (2003, chapter 7) and Casullo (2010). 
(C1) Conceivability provides epistemic access to the general modal status of propositions; or

(C2) Conceivability provides epistemic access to the specific modal status of propositions.

Hill's argument establishes that (C2) is false. But, from the fact that (C2) is false, it does not follow that $(\mathrm{C} 1)$ is false. Moreover, if the B-account of modal knowledge is correct, it follows that coherent conceiving is as reliable a guide to the general modal status of E-propositions as it is to the general modal status of AR-propositions.

Consider again

(P1) George W. Bush is a human being.

According to Hill, the following proposition can be known a priori

(P2) If George W. Bush is a human being then (ПQ) (Q> George W. Bush is a human being).

Moreover, (P2) follows from a more general a priori principle:

(21) $(\forall \mathrm{x})(\forall \mathrm{K})$ (if $\mathrm{x}$ is a biological substance and $\mathrm{K}$ is the biological kind to which $\mathrm{x}$ belongs, then (ПQ) ( $\mathrm{Q}>\mathrm{x}$ is a biological substance that belongs to $\mathrm{K}$ ),

which is partially constitutive of the subjunctive conditional. Since (21) is an a priori principle that is partially constitutive of the subjunctive conditional, it is an AR-proposition. Therefore, the proposition

(P7) It is not the case that if George W. Bush is a human being, then necessarily George W. Bush is a human being

is not coherently conceivable.

More generally, Hill maintains that, where $\mathrm{P}$ is any E-proposition, the corresponding proposition of the form

(25) If $\mathrm{P}$, then (ПQ) $(\mathrm{Q}>\mathrm{P})$

follows from more general principles that are partially constitutive of the subjunctive conditional and can be known a priori. Therefore, it is a consequence of the $\mathrm{B}$-account of modal knowledge that coherent conceiving is a reliable guide to 
the general modal status of E-propositions. Where $\mathrm{P}$ is any E-proposition, the corresponding proposition of the form

(P8) It is not the case that if $\mathrm{P}$, then it is metaphysically necessary that $\mathrm{P}$

is not coherently conceivable.

The B-account of modal knowledge has two consequences. First, coherent conceiving is a reliable guide to the general modal status of all necessary propositions. The access that it provides to the general modal status (or necessity) of E-propositions is as reliable as the access that it provides to the general modal status (or necessity) of AR-propositions. Second, although coherent conceiving is a reliable guide to the specific modal status of AR-propositions, it is not a reliable guide to the specific modal status of E-propositions. ${ }^{7}$ Hence, Hill is correct in maintaining that coherent conceiving is not a reliable guide to the specific modal status of E-propositions. However, since he fails to distinguish between the general modal status and the specific modal status of necessary propositions, he overlooks the fact that the principle that coherent conceiving is a reliable guide to the general modal status of E-propositions is a consequence of the B-account of modal knowledge. ${ }^{8}$

\section{REFERENCES}

Casullo, Albert. 2003. A Priori Justification. New York: Oxford University Press. . 2010. "Knowledge and Modality." Synthese 172: 341-359.

Hill, Christopher. 2006. "Modality, Modal Epistemology, and the Metaphysics of

Consciousness." In The Architecture of the Imagination, ed. S. Nichols. Oxford: Oxford University Press.

Kripke, Saul. 1971. "Identity and Necessity." In Identity and Individuation, ed. M. K. Munitz. New York: New York University Press.

Lewis, David. 1973. "Counterfactuals and Comparative Possibilities." Journal of Philosophical Logic 2: 418-446.

Williamson, Timothy. 2007. The Philosophy of Philosophy. Oxford: Blackwell.

7. Coherent conceiving is not a reliable guide to the specific modal status of E-propositions because one can coherently conceive of the negation of any E-proposition. Coherent conceiving is a reliable guide to the specific modal status of AR-propositions because one cannot coherently conceive of the negation of any AR-proposition since coherent conceiving requites compatibility with all AR-propositions.

8. Thanks to John Gibbons, Chris Hill, and Joe Mendola for their insightful comments on an earlier version of this essay. 